\title{
Der Tag der Kranken 2014 - ein Jubiläum
}

\author{
Immer am ersten Sonntag im März findet in der ganzen Schweiz der «Tag der Kran- \\ ken» statt - in diesem Jahr wird dies der 2. März 2014 sein. Auf 75 Bestehensjahre \\ kann zudem der gleichnamige Verein blicken. Das Motto des Jubiläumsjahres lautet: \\ «Psychische Belastungen: Zeigen wir Mut zum Dialog». Für Ärzte und Ärztinnen gibt \\ es dabei verschiedene Aspekte zu beleuchten.
}

Ursula Steiner-König

Delegierte der FMH ins Zentralkomitee des «Tag der Kranken»

Das Thema psychische Belastungen findet sich vielfach in den Medien. Dabei geht es beispielsweise um ADHS, Autismus und Aspergersyndrom, um Burnout oder Depression. Und doch nimmt die Stigmatisierung der Menschen mit psychischen Problemen oder gar Krankheiten nicht etwa ab, sondern eher noch zu. Das zeigt sich nicht zuletzt in der politischen Beurteilung dieser Problematik, mit deren Folgen sich Ärzte wie Patienten dann auseinandersetzen müssen.

So ist es äusserst bedenklich, wie etwa im Kanton Bern Sparmassnahmen gutgeheissen werden, die zur Folge haben, dass Psychischkranke ohne andere Plazierungsmöglichkeit «einfach» aus den Kliniken entlassen werden sollen, nachdem eine stationäre Klinikbehandlung nicht länger angezeigt ist. Es ist leider eine Tatsache, dass es viel zu wenig Institutionen gibt, die solche Patienten und Patientinnen aufneh-

\section{An Kranke denken, sie besuchen, sie bei chronischen}

\section{Erkrankungen nicht im Stich lassen.}

Korrespondenz:

Dr. med. Ursula Steiner-König Beim Goldenen Löwen 3

CH-4052 Basel

usteiner[at]hin.ch men und weiter betreuen könnten. Die Schliessung der Psychosomatischen Abteilung/Lory-Haus an der Universitätsklinik Inselspital ist aus ärztlicher Sicht ebenso wenig einleuchtend. Nicht nur, weil sie die einzige universitäre Einheit zur stationären Behandlung psychosomatisch Kranker ist, sondern weil ich den Eindruck habe, die Gesundheitspolitiker vergessen aufgrund der Diagnosen (die ihrer Meinung nach wohl nicht zum Katalog der einer Universitätsklinik würdigen Erkrankungen gehören), dass Menschen an diesen Diagnosen leiden. Das Leiden hat in der Ökonomie und Rationalisierung nichts verloren ...

Auch eine Situation aus dem Kanton Baselland habe ich als stossend wahrgenommen: Die zuständige Einbürgerungsbehörde erteilte zwar einer mehrköpfigen ausländischen Familie das Bürgerrecht, nicht aber dem einen Sohn. Er wurde davon ausgeschlossen, weil er nicht in der Lage war, dem Aufnahmeprozedere zu folgen. Er leidet unter einer schweren Schi- zophrenie, erfasst also Realitäten nicht wie andere normale Menschen. Den Politikern aber machte das mehrmals - trotz Einsprachen von Brüdern des Patienten - offenbar keinen Eindruck. «Wer nicht normal ist, hat kein Anrecht auf Einbürgerung», so dachten sie offenbar.

Denken wir schliesslich an die vielen in schier allen medizinischen Fachgebieten vorstellig werdenden Kranken mit somatoformen Schmerzstörungen, die von der Invalidenversicherung nicht als krank und leistungsberechtigt angesehen werden. Auch begutachtende Ärzte neigen dazu, diesen Patientinnen und Patienten mehr zuzumuten, als deren Hausärzte raten. Während der behandelnde Arzt diese Menschen über lange Zeit begleitet und ihreErwerbsfähigkeit nicht selten anders einschätzt, sieht sich der Gutachter vor die Aufgabe gestellt, sich aufgrund einer punktuellen Untersuchung ein Bild über Leistungsfähigkeit und Zumutbarkeit zu machen. Dies mit dem Hintergrundwissen, dass möglichst wenig neue IV-Renten oder andere IV-Leistungen gesprochen werden sollten. Keine beneidenswerte Sandwich-Stellung.

Wenn wir zudem bedenken, dass die Anzahl der Weiterbildungskandidaten in Psychiatrie und Psychotherapie enorm abgenommen hat, sodass in den kommenden Jahren für viele psychiatrisch-psychotherapeutische Privatpraxen kaum Chancen bestehen, durch eine schweizerische Nachfolge neubesetzt zu werden, so sind heute alle Ärztinnen und Ärzte aufgerufen, Mut zum Dialog zu zeigen. Sie alle können die eigenen Patientinnen und Patienten möglichst offen auf allfällig spürbare psychische Belastungen ansprechen und ihnen damit wenigstens einen Weg zu weiteren Behandlungsschritten eröffnen.

Zum Glück gibt es eine stattliche Anzahl von ausländischen Kolleginnen und Kollegen, die das schweizerische Weiterbildungs-Curriculum für Psychiatrie und Psychotherapie absolvieren. Es ist qualitativ mit Abstand besser als manches ausländische, als gleichwertig EU-anerkanntes. Wir wissen auch, dass in den nächsten Jahren viele psychologisch ausgebildete Fachpsychotherapeuten den Markt erweitern werden. Wie begabt Letztere im Verstehen psychosomatischer Phänomene sein werden, steht hier nicht zur Diskus- 
sion. Fakt ist: Es ist das Vertrauensverhältnis zwischen Patient und Arzt, das den Ausschlag gibt, weit mehr als Theorie und Wissen einer Fachperson.

Natürlich braucht es abgesehen vom Verständnis für psychische Belastungen auch Fachwissen. Dieses kann nicht ubiquitär vorausgesetzt werden. Gute interdisziplinäre Zusammenarbeit sollte daher selbstverständlich sein. Als Fachfrau «auf dem Lande» habe ich glücklicherweise viele gute Erfahrungen mit solcher Zusammenarbeit machen können, wenn es sich auch gelegentlich zeigte, dass eigene Gärtlein gern gehütet werden wollten. Apropos «Mut zum Dia$\log$ »: Ich möchte an dieser Stelle nicht wiederholen, was dem Faktenblatt des «Tag der Kranken» unter www.tagderkranken.ch entnommen werden kann, lediglich für uns Ärztinnen und Ärzte ergänzen: Zeigen wir auch Mut zum interdisziplinären Dialog. Denn er kommt nicht nur uns, sondern vor allem unseren Patientinnen und Patienten zugute und muss dabei nicht zwingend kostentreibend sein.

\section{Jahre Tag der Kranken}

An Kranke ganz allgemein zu denken, sie zu besuchen, sie etwa bei chronischen Erkrankungen nicht im Stich zu lassen: Das soll ja nicht nur für den 1. Sonntag im März von Bedeutung sein, sondern fürs ganze Jahr gelten. Die Gründerin des «Tag der Kranken», Dr. med. Marthe Nicati, war selber in Leysin vorwiegend mit Tuberkulosekranken beschäftigt und wollte mit der Gründung des Vereins Folgendes erreichen:

- gute Beziehungen zwischen Gesunden und Kranken fördern;
- an Pflichten der Gesunden den Kranken gegenüber erinnern;

- das Verständnis für Bedürfnisse der Kranken fördern;

- die Tätigkeit all jener anerkennen, die sich beruflich oder privat für Patienten einsetzen.

Marthe Nicati hatte die Vereinsamung ihrer Sanatoriums-Patientinnen und -Patienten erkannt und wollte etwas dagegen unternehmen. Ihre Anliegen haben - trotz grossem Wandel in der Medizin - bis heute nicht an Bedeutung verloren. Das Zentralkomitee des «Tag der Kranken» freut sich, dass seine Institution bereits das Alter von einem Dreiviertel-Jahrhundert erreicht hat und wünscht sich, dass sie noch lange weiterlebe!

\section{Zum Jubiläum: Karten und ein Plakat}

Als besonderes Zeichen des Jubiläums und als kleine Aufmerksamkeit, die das ganze Jahr über eingesetzt werden kann, hat uns ANNA eine Karte entworfen, auf deren Rückseite bereits Genesungswünsche vorgedruckt sind.

Zudem gibt es kleine Plakate. Sie können von jeder Kollegin, jedem Kollegen beim «Tag der Kranken» www.tagderkranken.ch heruntergeladen, Karten in drei Sprachen auf der Homepage angeschaut und entsprechend bestellt werden.

Ein kleines Plakat im Wartezimmer, als Ausdruck unserer Solidarität, wäre sehr willkommen!

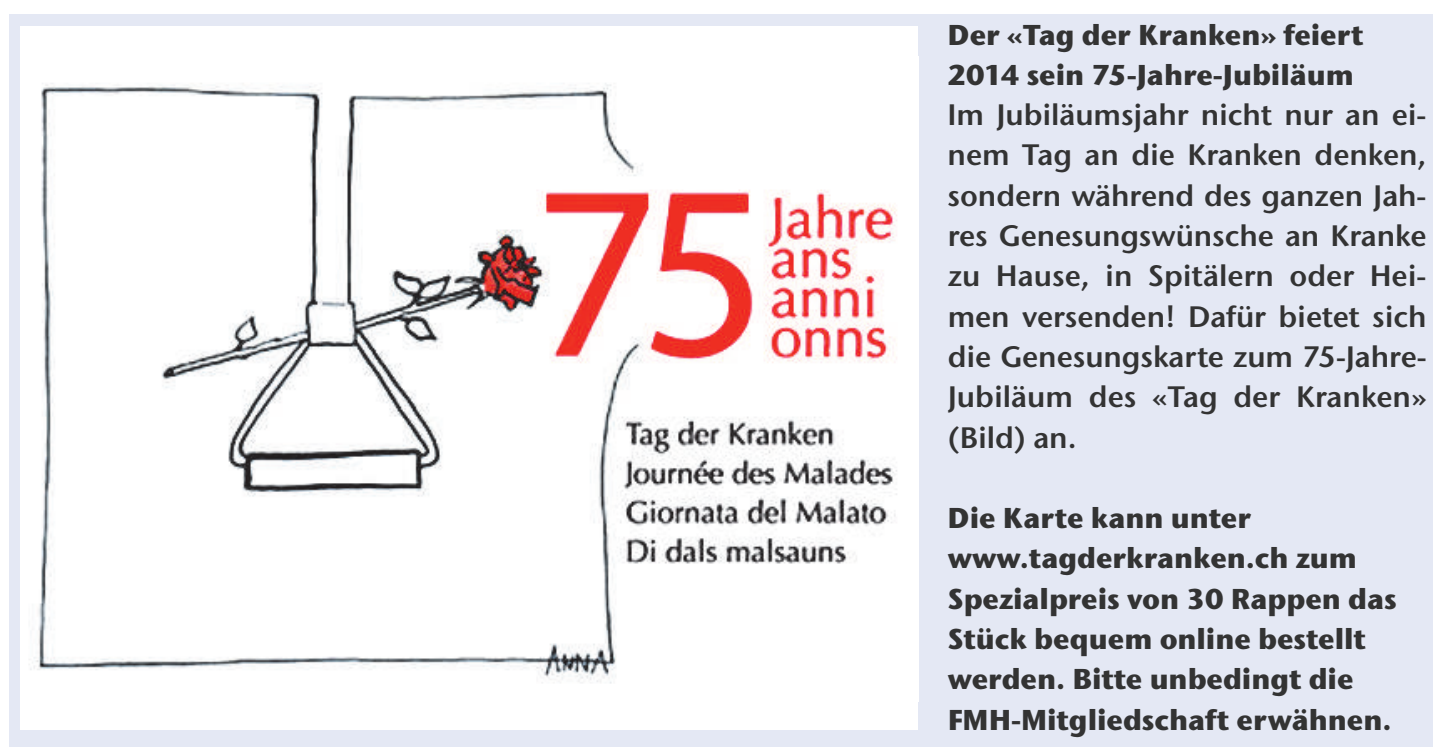

